\title{
A Numerical Determination of the Modulus of Doubly Connected Domains by Using the Bergman Curvature
}

\author{
By J. Burbea*
}

\begin{abstract}
The moduli of doubly connected domains are computed by means of the Bergman curvature associated with the Bergman function of the domain. The moduli of nonconcentric annuli, elliptic rings, confocal elliptic rings, squares inside circles and squares inside squares are determined by this method.
\end{abstract}

1. Introduction. The moduli of doubly connected domains are computed by means of the curvature associated with the Bergman kernel function of the domain. The method is based on the fact that the curvature assumes a minimal value only on a distinguished unique curve in the domain. This curve is the conformal pre-image of the circle $|z|=\sqrt{ } r$ which lies in the annulus $\{z: r<|z|<1\}, 0<r<1$, where $r$ is the modulus of the given doubly connected domain.** Further, because of the conformal invariance of the curvature, its minimal value on the distinguished curve must coincide with the curvature of the annulus evaluated on the circle $|z|=\sqrt{ } r$. This gives us at once an effective method to determine the modulus $r$ of the given doubly connected. domain.

As an example of this method, we determine the moduli of nonconcentric annuli, elliptic rings, confocal elliptic rings, squares inside circles and squares inside squares, and compare numerical results to known data. We also compute and analyze the behavior of the curvature in the annulus. The computation is programmed in Fortran and run on the Golem-1 and CDC 1604A at the Weizmann Institute of Science, Rehovot, Israel. In general, the method gives good accuracy. For fourfold symmetric domains the method can be simplified to decrease the amount of computation needed to yield the same accuracy as would be achieved by the method for the general case.

2. General Theory. Let $D$ be a bounded finitely connected domain in the $z$-plane. The theory of Hilbert spaces with reproducing kernels allows us to introduce the Bergman kernel function $K_{D}(z, \bar{t}), z, t \in D . K_{D}(z, \bar{t})$ is uniquely determined as the reproducing kernel of the space of all square integrable analytic functions in $D$, $\mathcal{L}^{2} H(D)$. As such [1, p. 9], the Bergman kernel function is analytic in $z \in D$ ( $t$ fixed) and anti-analytic in $t \in D$ ( $z$ fixed). It is more convenient, especially when the connectivity of $D$ is greater than one, to restrict ourselves to the subspace of $\mathscr{L}^{2} H(D)$, $\mathfrak{L}_{\mathrm{s}}^{2} H(D)$, which consists of all functions of $\mathfrak{L}^{2} H(D)$ which have a single valued indefinite integral. This subspace is closed and, thus, it is also a Hilbert space with the

Received April 30, 1970, revised March 29, 1971.

AMS 1969 subject classifications. Primary 6510, 6520.

Key words and phrases. Bergman curvature, Bergman kernel, Bergman metric, conformal invariance, modulus, reproducing kernel, Szegö kernel, theorema egregium.

* This work was supported in part by Contract AEC 326-P22 at Stanford University.

** Frequently in the literature $1 / r$ appears as the modulus of the domain. 
reduced Bergman kernel $K_{D}^{(s)}(z, \bar{t})$. Henceforth, we simply write, unless otherwise specified, $K_{D}(z, \bar{t})$ instead of $K_{D}^{(s)}(z, \bar{t})$ and $\mathscr{L}^{2} H(D)$ instead of $\mathscr{L}_{a}^{2} H(D)$.

Let $\left\{\Phi_{\nu}(z)\right\}, \nu=1,2, \cdots$, be a complete orthonormal sequence of functions in $\mathfrak{L}^{2} \equiv \mathscr{L}^{2} H(D)$, that is,

$$
\left(\Phi_{\mu}, \Phi_{\nu}\right) \equiv \int_{D} \Phi_{\mu}(z) \overline{\Phi_{\nu}(z)} d x d y=\delta_{\mu \nu}
$$

Then the kernel $K_{D}(z, \bar{t})$ is given by

$$
K_{D}(z, \bar{t})=\sum_{\nu=1}^{\infty} \Phi_{\nu}(z) \overline{\Phi_{\nu}(t)}
$$

This sum is independent of the choice of the complete orthonormal system and it converges absolutely and uniformly on each compact subset of $D$.

We introduce the Bergman metric

$$
d S_{D}^{2}=K_{D}(z, \bar{z})|d z|^{2},
$$

which is invariant under conformal mapping. The Gaussian curvature of this metric is given by

$$
C_{D}(z)=\frac{-2}{K} \frac{\partial^{2} \log K}{\partial z \partial \bar{z}}, \quad K \equiv K_{D}(z, \bar{z}) .
$$

We refer to this curvature as the Bergman curvature. It has the following important properties:

(i) $C_{D}(z)$ is strictly negative;

(ii) $C_{D}(z)$ is invariant under conformal mapping;

(iii) $C_{D}(z)$ has boundary values $-4 \pi$ (see $[1$, p. 39]).

Property (ii) can be proved directly from the fact that (2.3) is a conformal invariant or by the use of the "theorema egregium" of Gauss.

From property (ii) and the Riemann mapping theorem, it follows that $C_{D}(z)$ is a universal constant for all simply connected domains $D$, and, in fact, $[1$, p. 35]

$$
C_{D}(z) \equiv-4 \pi, \quad D \text { simply connected domain. }
$$

It will be shown later that if $D$ is doubly connected, then $C_{D}(z)$ is not a constant. The function $C_{D}(z)$ can be used to determine whether two domains, say $D$ and $D^{*}$, can be mapped conformally onto each other. If this is the case, one can also construct the mapping function.

We introduce the quantity

$$
J_{D}(z)=-\frac{1}{2} C_{D}(z)
$$

or, by (2.4),

$$
J_{D}(z)=\frac{1}{K} \frac{\partial^{2} \log K}{\partial z \partial \bar{z}}, \quad K \equiv K_{D}(z, \bar{z}) .
$$

We conclude this section by enumerating the properties of $J_{D}(z)$ corresponding to properties (i), (ii) and (iii) of $C_{D}(z)$ in the following theorem:

THEOREM 2.1. Let $D$ be a domain in the z-plane, then

(i) $J_{D}(z)$ is strictly positive;

(ii) $J_{D}(z)$ has boundary values $2 \pi$. 
Let $w=w(z)$ be a conformal mapping which maps $D$ onto the domain $D^{*}$ in the w-plane, then

$$
d S_{D}^{2}=d S_{D^{*}}^{2}, \quad J_{D}(z)=J_{D^{*}}(w)
$$

3. The Modulus of a Doubly Connected Domain. Let $D$ be a doubly connected domain in the $w$-plane bounded by two Jordan curves $C_{1}$ and $C_{2}$ for which $C_{1} \cap C_{2}=\varnothing$. As is well known, $D$ can always be mapped conformally onto the annular domain,

$$
R=\{z: r<|z|<1\}, \quad 0<r<1 .
$$

The number $r$ is called the "modulus of $D$ " and it characterizes completely the conformal equivalence class of $D$. Furthermore, since the only conformal mappings of $R$ onto itself are of the form $\varphi(z)=\gamma z$ or $\varphi(z)=\gamma r / z$, where $|\gamma|=1$, it follows that if $w=w(z)$ furnishes one particular mapping of $D$ onto $R$, then all conformal mappings of $D$ onto $R$ are of the form $\varphi(z)=\gamma w(z)$ and $\varphi(z)=\gamma r / w(z)$. Thus, given a point $t \in D$, the set of all its possible images consists of precisely two circles (which degenerate to one circle if $|w(t)|=\sqrt{ } r)$, namely $|\varphi|=|w(t)|,|\varphi|=r /|w(t)|(\varphi=\varphi(z))$.

Let $J(z)=J_{R}(z)$, since $R$ can be mapped conformally onto itself in such a manner that the points $t, \tau \in R$ will correspond to each other whenever $|t|=|\tau|$ or $|t|=r /|\tau|$, it follows that $J(z)$ (or any other conformal invariant) must be a constant along each circle $|z|=A, A$ constant, and the constant values associated with the pair of circles $|z|=A$ and $|z|=r / A$ must coincide. Thus, we can write

$$
J(z)=J(|z|)=J(r /|z|), \quad z \in R .
$$

Equivalently, if $\rho^{2}=z \bar{z}$, then,

$$
J(z)=J(\rho)=J(r / \rho), \quad r<\rho<1 .
$$

By a detailed use of the theory of elliptic functions [12, pp. 101-102], [1, p. 41], one can show the following important theorem.

THEOREM 3.1. In the annulus $R, J(z)$ is a positive function of $\rho=|z|, \rho \in[r, 1]$ satisfying (3.3). Furthermore, $J(\rho)$ has only one maximum at $\rho=\sqrt{ } r$, while

$$
J(1)=J(r)=2 \pi .
$$

It should be emphasized that the use of the "reduced" Bergman kernel function is very essential in the proof of Theorem 3.1, since the theorem is not true for the usual Bergman kernel function. It could be mentioned, however, that a similar theory for determining the modulus of a doubly connected domain, by using the usual Szegö kernel function, holds (see [4]) and yields the same results.

Next, it can be shown that the maximum value $J(\sqrt{ } r)$ is itself a monotone function of $r$. This gives us at once an effective method to determine the modulus of any doubly connected domain $D$. First, we determine the modulus $r$ of $D$ by solving the implicit equation

$$
J(\sqrt{ } r)=\max _{z \in D} J_{D}(z) .
$$

Since $J(\sqrt{ } r)$ is monotone in $r,(3.5)$ has a unique solution $r$, which is the modulus of $D$. Furthermore, since $J_{D}(z)$ is not constant, by determining its level lines and their orthogonal trajectories, we can find numerically the mapping function. 
4. The Numerical Algorithm. Let $D$ be a doubly connected domain in the $z$-plane bounded by the Jordan curves $C_{1}$ and $C_{2}\left(C_{1} \cap C_{2}=\varnothing\right)$ and suppose the origin lies in the "hole" of $D$. As is well known, the set $\left\{z^{n}\right\}_{n=-\infty}^{\infty}, n \neq-1$, forms a complete set in $\mathscr{L}^{2} H(D)$ if $D$ separates 0 from $\infty$. By applying the Gram-Schmidt procedure, we obtain the sequence of orthonormal functions $\left\{P_{n}(z)\right\}_{-\infty}^{\infty}, n \neq-1$. For this purpose, it is more convenient to reorder the powers $\left\{z^{n}\right\}_{n=-\infty}^{\infty}, n \neq-1$, by the following rule

$$
\beta_{1}=1, \quad \beta_{2}=z, \quad \beta_{n}=z^{(-1)^{n+1}[(n+1) / 2]}, \quad n>2 .
$$

Then, the complete orthonormal sequence of functions $\left\{P_{n}(z)\right\}_{n=1}^{\infty}$ is given by

$$
P_{n}(z)=\sum_{k=1}^{n} a_{n k} \beta_{k}, \quad n=1,2, \cdots .
$$

The $\left\{a_{n k}\right\}$ are determined recursively by

$$
a_{n k}=c_{n k} /\left(\sum_{i=1}^{n} \sum_{i=1}^{n} c_{n i} \bar{c}_{n i}\left(\beta_{i}, \beta_{i}\right)\right)^{1 / 2}, \quad k=1, \cdots, n ; n=1,2, \cdots,
$$

where

$$
c_{n n}=1 ; \quad c_{n k}=-\sum_{i=k}^{n-1} a_{i k}\left(\sum_{j=1}^{i} \bar{a}_{i i}\left(\beta_{n}, \beta_{i}\right)\right), \quad k=1,2, \cdots, n-1 .
$$

We can use Green's formula to evaluate the moments

$$
\begin{aligned}
\left(\beta_{m}, \beta_{n}\right)=\int_{D} \beta_{m} \bar{\beta}_{n} d x d y=\frac{1}{2 i n} \int_{C} \beta_{m} \bar{z}^{n} d z, \quad n=1,2, \\
\left(\beta_{m}, \beta_{n}\right)=\int_{D} \beta_{m} \bar{\beta}_{n} d x d y \\
=\frac{1}{2 i\left\{(-1)^{n+1}[(n+1) / 2]+1\right\}} \int_{C} \beta_{m} \bar{z}^{(-1)^{n+1}[(n+1) / 2]+1} d z, \quad n>2,
\end{aligned}
$$

where

$$
C=\partial D=C_{1} \cup C_{2} .
$$

The kernel function is then given by

$$
K_{D}(z, \bar{z})=\sum_{n=1}^{\infty} P_{n}(z) \overline{P_{n}(z)},
$$

while, by (2.7),

$$
J_{D}(z)=\frac{1}{K^{3}}\left[K K_{z \bar{z}}-K_{z} K_{\bar{z}}\right], \quad K \equiv K_{D}(z, \bar{z}),
$$

where

$$
K_{z}=\sum_{n=1}^{\infty} P_{n}^{\prime}(z) \overline{P_{n}(z)}, \quad K_{\bar{z}}=\sum_{n=1}^{\infty} P_{n}(z) \overline{P_{n}^{\prime}(z)}, \quad K_{z \bar{z}}=\sum_{n=1}^{\infty} P_{n}^{\prime}(z) \overline{P_{n}^{\prime}(z)} .
$$

It can be shown that

$$
J(\sqrt{ } r)=\frac{1}{r}+\frac{1}{r} \sum_{n=1}^{\infty} \frac{n^{3} r^{n}}{1-r^{2 n}} / \sum_{n=1}^{\infty} \frac{n r^{n}}{1-r^{2 n}},
$$


and further, that this quantity is monotonically decreasing in $r, 0<r<1$. The modulus $r$ is the unique solution of the equation

$$
\max _{z \in D} J_{D}(z)=J(\sqrt{ } r) .
$$

The computational method is as follows. The doubly connected domain $D$ is given by its boundary $C=C_{1} \cup C_{2},\left(C_{1} \cap C_{2}=\varnothing\right)$, which is described by the set of $2 M$ complex numbers $z_{\nu k}=x_{\nu k}+i y_{\nu k}, z_{\nu k} \in C_{\nu}, \nu=1,2 ; k=1,2, \cdots, M$. Numerically, the moments (4.5) and (4.6) are approximated by finite sums of $M$ terms "balanced" with conveniently chosen positive weights, that is,

$$
\left(\beta_{m}, \beta_{n}\right)=\frac{1}{2 i n}\left[F_{1}(M ; m, n)-F_{2}(M ; m, n)\right], \quad n=1,2,
$$

where,

$$
F_{\nu}(M ; m, n)=\sum_{k=1}^{M} w_{k}^{(\nu)} z_{\nu k}^{(-1)^{m+1}[(m+1) / 2]} \bar{z}_{\nu k}^{n}, \quad \nu=1,2 ; m>2,
$$

and

$$
F_{\nu}(M ; m, n)=\sum_{k=1}^{M} w_{k}^{(v)} z_{v k}^{m-1} \bar{z}_{\nu k}^{n}, \quad \nu=1,2 ; m=1,2
$$

Next,

$$
\begin{array}{r}
\left(\beta_{m}, \beta_{n}\right)=\frac{1}{2 i\left\{(-1)^{n+1}[(n+1) / 2]+1\right\}}\left[G_{1}(M ; m, n)-G_{2}(M ; m, n)\right] \\
n>2
\end{array}
$$

where

$$
\begin{aligned}
& G_{\nu}(M ; m, n)=\sum_{k=1}^{M} w_{k}^{(\nu)} z_{\nu k}^{(-1)^{m+1}[(m+1) / 2]} \bar{z}_{\nu k}^{(-1))^{n+1}[(n+1) / 2]+1}, \\
& \nu=1,2 ; m>2 \text {, }
\end{aligned}
$$

and

$$
G_{\nu}(M ; m, n)=\sum_{k=1}^{M} w_{k}^{(\nu)} z_{\nu k}^{m-1} \bar{z}_{\nu k}^{(-1)^{n+1}[(n+1) / 2]+1}, \quad \nu=1,2 ; m=1,2 .
$$

Here, $w_{k}^{(v)}, \nu=1,2 ; k=1,2, \cdots, M$, are the positive weights chosen such that the finite sums approximate the integrals over $C$. Then, using (4.3) and (4.4), we obtain an approximation of $\left\{P_{k}(z)\right\}_{k=1}^{n}$, see (4.2), whence we form the sum

$$
K_{n}(z, \bar{z})=\sum_{k=1}^{n} P_{k}(z) \overline{P_{k}(z)}
$$

After formal differentiation of the $\left\{P_{k}(z)\right\}$, we obtain the quantity $J_{n}(z)$, (here $\left.\lim _{n \rightarrow \infty} J_{n}(z)=J_{D}(z)\right)$, see (4.9) and (4.10).

Specifically, we assume that the two bounding curves $C_{v}, \nu=1,2$, are starshaped with respect to $z=0{ }^{* * *}$ Next, we choose $2 N$ complex numbers $\zeta_{\nu k} \in C_{\nu}$ such that

*** A modified method can be given if the two bounding curves are not starshaped. 


$$
\zeta_{\nu k}=\rho_{\nu k} e^{i \theta_{k}}, \quad \nu=1,2 ; k=1,2, \cdots, N,
$$

and then we form the real functions

$$
g_{k}^{(n)}(\rho)=J_{n}\left(\rho e^{i \theta_{k}}\right), \quad \rho \in\left[\rho_{2 k}, \rho_{1 k}\right], \quad k=1,2, \cdots, N .
$$

On the interval $\left[\rho_{2 k}, \rho_{1 k}\right], g_{k}^{(n)}(\rho)$ are real and positive functions such that

$$
\lim _{n \rightarrow \infty} g_{k}^{(n)}\left(\rho_{\nu k}\right)=2 \pi, \quad \nu=1,2 ; k=1, \cdots, N .
$$

Formula (4.22) is interpreted as follows:

$$
\begin{aligned}
\lim _{\rho \rightarrow \rho, k} \lim _{n \rightarrow \infty} g_{k}^{(n)}(\rho) & =\lim _{\rho \rightarrow \rho, k} \lim _{n \rightarrow \infty} J_{n}\left(\rho e^{i \theta_{k}}\right) \\
& =\lim _{\rho \rightarrow \rho_{, k}} J_{D}\left(\rho e^{i \theta_{k}}\right) \equiv J_{D}\left(\rho_{\nu k} e^{i \theta_{k}}\right)=2 \pi .
\end{aligned}
$$

Theoretically, this convergence always takes place if $D$ is assumed to ${ }_{\alpha}$ have the property that through $\zeta_{\nu k}(\nu=1,2 ; k=1,2, \cdots, N)$ two circles can be constructed in such a way that one circle is interior to $D$ and the second is exterior to $D$. Clearly, our assumptions on $D$ satisfy the above requirements. Moreover, by [1, p. 39], (4.22) will be true if

$$
\left|\arg \left(\rho e^{i \theta_{k}}-\rho_{\nu k} e^{i \theta_{k}}\right)\right| \leqq \alpha<\frac{\pi}{2}+\left|\theta_{k}\right|,
$$

which is trivially valid as long as the approach is along the line $\rho e^{i \theta_{k}}$. We first find the maximal points of $g_{k}^{(n)}(\rho)$ by the Fibonacci search (see [8], [10]). ${ }^{\dagger}$ Consequently, we obtain the quantities

$$
g_{k}^{(n)}\left(\rho_{k}\right)=\max g_{k}^{(n)}(\rho), \quad \rho \in\left[\rho_{2 k}, \rho_{1 k}\right], \quad k=1,2, \cdots, N .
$$

Next, we form

$$
g_{m}^{(n)}\left(\rho_{m}\right)=\max g_{k}^{(n)}\left(\rho_{k}\right), \quad k=1,2, \cdots, N, \quad(1 \leqq m \leqq N) .
$$

We also truncate $J(\sqrt{ } r)$ in (4.11) to obtain

$$
J_{n}(\sqrt{ } r)=\frac{1}{r}+\frac{1}{r} \sum_{k=1}^{n} \frac{k^{3} r^{k}}{1-r^{2 k}} / \sum_{k=1}^{n} \frac{k r^{k}}{1-r^{2 k}} .
$$

Consider

$$
S(r)=J_{n}(\sqrt{ } r)-g_{m}^{(n)}\left(\rho_{m}\right) .
$$

The approximate modulus $r$ of $D$ is determined from the implicit equation

$$
S(r)=0, \quad 0<r<1 .
$$

Numerically, we find the solution of (4.27) by the "Newton-Raphson iteration method." The quality of the approximation and the rate of convergence is dependent on the number of the boundary points $2 N$ and the number of functions $n$ of $\left\{\beta_{i}\right\}_{i=1}^{n}$ actually used in the orthonormalization.

\footnotetext{
${ }^{\dagger}$ It should be remarked that, in order to apply this method, one has to verify that every level curve is starshaped with respect to $z=0$ provided that two bounding curves $C_{\nu}(\nu=1,2)$ are so. This is, however, a simple consequence of the minimum principle for harmonic functions, which, for completeness, will be included in the Appendix of this paper.
} 
5. Fourfold Symmetric Domains. Let $D$ be a doubly connected fourfold symmetric domain around the origin. Suppose again the boundary of $D$ is given by $C=C_{1} \cup C_{2},\left(C_{1} \cap C_{2}=\varnothing\right)$. Because of the fourfold symmetry, the boundary components $C_{\eta}, \nu=1,2$, are composed of four symmetrical parts

$$
C_{\nu}=\bigcup_{k=1}^{4} C_{v k}, \quad \nu=1,2 .
$$

The moments $\left(z^{m}, z^{n}\right)$ are given by

$$
\begin{array}{rlrl}
\left(z^{m}, z^{n}\right) & =0, & m-n & \neq 0(\bmod 2), \\
& =\frac{2}{n+1}\left(A_{11}-A_{21}\right), & m-n \equiv 0(\bmod 2),
\end{array}
$$

where

$$
A_{\nu 1}=\int_{C, 1}|z|^{2 m} \operatorname{Im}\left[\bar{z}^{n-m+1}\right] d z, \quad \nu=1,2 .
$$

When the powers $\left\{z^{n}\right\}_{n=-\infty}^{\infty}, n \neq-1$, are reordered according to the rule (4.1), we obtain the complete orthonormal set of functions $\left\{P_{n}(z)\right\}_{n=1}^{\infty}$ as given in (4.2)-(4.4); however,

$$
\begin{aligned}
& a_{m n}=\bar{a}_{m n}, \quad c_{m n}=\bar{c}_{m n}, \quad m-n \equiv 0(\bmod 2), \\
& a_{m n}=c_{m n}=0, \quad m-n \not \equiv 0(\bmod 2) .
\end{aligned}
$$

Therefore, $\overline{P_{n}(z)}=P_{n}(\bar{z})$ and, by (4.19),

$$
K_{n}(z, \bar{z})=\sum_{k=1}^{n} P_{k}(z) P_{k}(\bar{z})
$$

We obtain the following significant simplification. First, "half" of the moments needed for the orthonormalization vanish, and second, the remaining moments are real. Therefore, the amount of computation needed for the orthonormalization is decreased significantly as compared to the case where the domain is not fourfold symmetric.

Numerically, we again use the scheme described in (4.13)-(4.18) to evaluate the moments $\left(\beta_{m}, \beta_{n}\right)$ where, by (5.2) and (5.3),

$$
A_{\eta 1}=\int_{C_{\eta 1}} \operatorname{Im}\left[z^{m} \bar{z}^{n+1}\right] d x+\int_{C_{\eta 1}} \operatorname{Re}\left[z^{m} \bar{z}^{n+1}\right] d y, \quad \nu=1,2 .
$$

Furthermore, because of the fourfold symmetry, we actually take $N_{1}=[N / 4]+1$ boundary points instead of $N$.

6. Numerical Results. We compute the moduli of some doubly connected domains and compare the numerical results to some known data. The computation is done according to Sections 4 and 5.

The results are presented in the following six examples. Each example is followed by several remarks relevant to that example. Especially, we mention in which way we evaluated the moments (4.5) and (4.6); however, wherever possible (as in the case of Examples 1, 2 and 5), we also exploit the analytic simplicity of the circular contours involved. First, we consider the following simple example. 
Example 1. Consider the concentric annulus

$$
R=\{z: 0.2<|z|<1\} .
$$

Define

$$
I(z)=\frac{1}{\pi} J_{R}(z)
$$

We evaluate $I(z)$ as a function of $|z|$, see (3.2) and (3.3), and see where the maximum occurs in $[0.2,1]$, see Table 1. Here, $J_{R}(z)$ is evaluated with the aid of (2.2) and (2.7). Observe that for the special case $D=R$ the powers $\left\{z^{n}\right\}_{n=-\infty}^{\infty}, n \neq-1$, are already orthogonal.

\section{TABLE 1}

\begin{tabular}{lc||lc}
\hline \multicolumn{1}{l||}{$|z|$} & $I(z)$ & $|z|$ & $I(z)$ \\
\hline 0.2 & 2.00000 & 0.5 & 4.03397 \\
0.222 & 2.00258 & 0.6 & 2.78008 \\
0.25 & 2.02576 & 0.7 & 2.40292 \\
0.333 & 2.78007 & 0.75 & 2.21434 \\
0.4 & 4.03397 & 0.8 & 2.02576 \\
0.45 & 4.40297 & 0.9 & 2.00257 \\
$\sqrt{0.2}$ & 4.40362 & 1.0 & 2.00000 \\
\hline
\end{tabular}

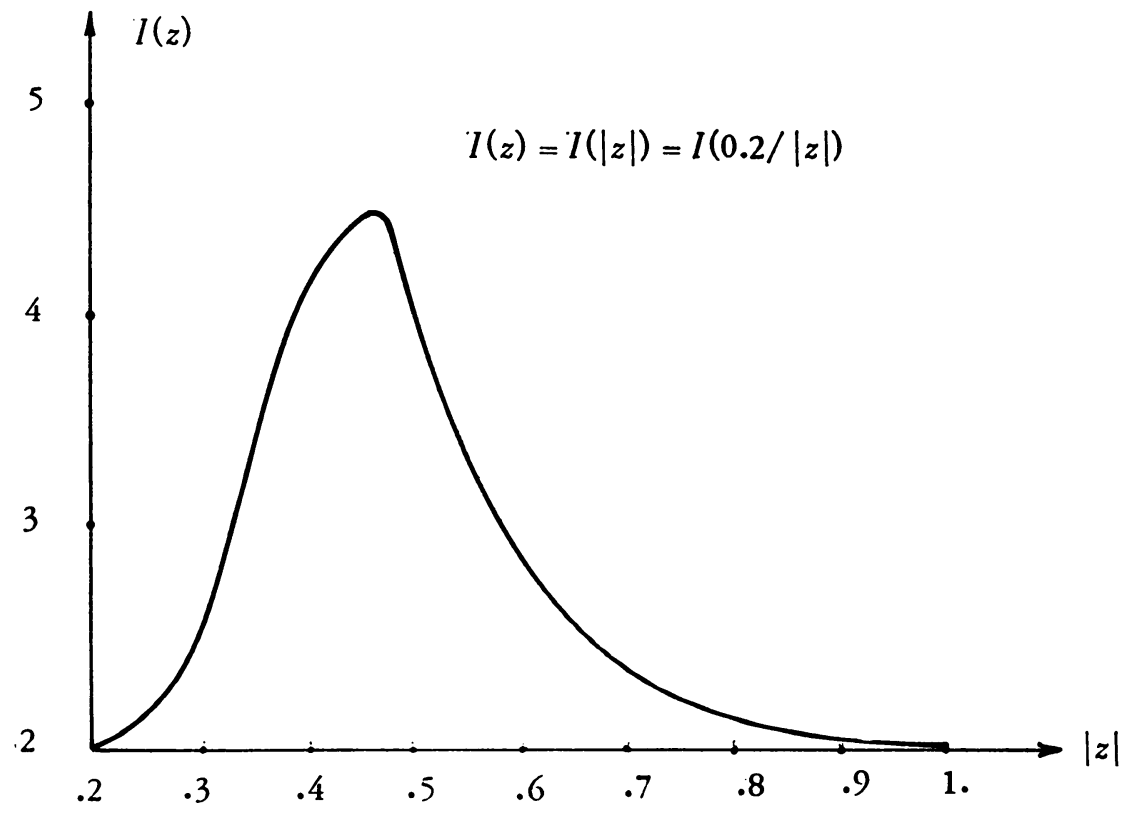

FIGURE 1 
As is seen from Table 1 and Fig. 1 , the maximum of $I(z)$ occurs at $|z|=(0.2)^{1 / 2}$, in agreement with Theorem 3.1, especially (3.4) is also satisfied here.

Example 2. Let $D$ be a nonconcentric annulus

$$
D=\{z:|z|<\rho\} \cap\left\{z:|z-m|>\rho_{1}\right\}, \quad \rho, m, \rho_{1}>0, \quad \rho>m+\rho_{1} .
$$

The function

$$
f(z)=(a z+\rho) /(z+a \rho),
$$

where

$$
a=(2 m \rho)^{-1}\left\{\left(\rho_{1}^{2}-\rho^{2}-m^{2}\right)-\left(\left(\rho_{1}^{2}-\rho^{2}-m^{2}\right)^{2}-4 m^{2} \rho^{2}\right)^{1 / 2}\right\},
$$

maps $D$ onto the annulus $R$ in (3.1). The modulus of $D$ is

$$
r=(a m+\rho) / \rho_{1} \text {. }
$$

If $\rho=8, \rho_{1}=2, m=4$, then $a=-1.8279344$ and $r=0.3441312$. Table 2 describes the results of our method. The moments here are computed analytically. Here, $2 n$ is the number of functions $\left\{z^{k}\right\}_{k--n}^{n}, k \neq-1$, needed for the orthonormalization and $2 N$ is the number of the boundary points taken in the computation.

TABLE 2

\begin{tabular}{rc|c}
\hline$n$ & $N$ & $r$ \\
\hline 8 & 5 & 0.3458634245 \\
8 & 9 & 0.3441189642 \\
12 & 5 & 0.3442653716 \\
12 & 9 & 0.3441296234 \\
16 & 5 & 0.3441744523 \\
16 & 9 & 0.3441308326 \\
\hline
\end{tabular}

As is shown in Table 2, the pair $(n, N)=(16,9)$ gives the closest result to the theoretical value $r=0.3441312$.

We consider fourfold symmetric domains in the following examples.

Example 3. Let $D$ be an elliptic ring

$$
D=E_{1} \cap E_{2}^{\prime},
$$

where

$$
E_{k}=\left\{(x, y): b_{k}^{2} x^{2}+a_{k}^{2} y^{2} \leqq a_{k}^{2} b_{k}^{2}\right\}, \quad k=1,2,
$$

and $E_{2}^{\prime}$ is the set theoretical complement of $E_{2}$. We also assume

$$
b_{2}<a_{2}<a_{1}, \quad b_{2}<b_{1}<a_{1} .
$$

Especially, let

$$
a_{1}=1.2, \quad a_{2}=0.3 ; \quad b_{1}=1, \quad b_{2}=0.2 .
$$

The approximated modulus of $D$ is given in Table 3 . 
Here, the moments are computed according to (5.2) and (5.7). We approximate

$$
A_{p 1} \cong \sum_{k=1}^{M} x_{k}^{(v)} \operatorname{Im}\left[z_{v k}^{m} \bar{z}_{v k}^{n+1}\right]+\sum_{k=1}^{M} y_{k}^{(v)} \operatorname{Re}\left[z_{v k}^{m} \bar{z}_{v k}^{n+1}\right], \quad \nu=1,2
$$

Here, $M=46$ and

$$
\begin{array}{rlrl}
z_{\nu k} & =a_{\nu} \cos \theta_{k}+i b_{\nu} \sin \theta_{k} \quad(\nu=1,2 ; k=1,2, \cdots, 46), \\
\theta_{k} & =(k-1) \pi / 90, & k & =1,2, \cdots, 46, \\
x_{k}^{(v)} & =-a_{v} \sin \theta_{k}, & \nu & =1,2, \\
y_{k}^{(v)} & =b_{\nu} \cos \theta_{k}, & \nu & =1,2 .
\end{array}
$$

TABLE 3

\begin{tabular}{rcc}
\hline$n$ & $N$ & $r$ \\
\hline 8 & 5 & 0.23234507814 \\
8 & 9 & 0.22965682330 \\
12 & 5 & 0.23388558847 \\
12 & 9 & 0.23087526739 \\
\hline
\end{tabular}

In the case of Table 3, the theoretical value of $r$ is not known, however, it is conceivable that the pair $(n, N)=(12,9)$ gives the closest result to the modulus of $D$. From Tables 2 and 3 , we see that the number of boundary points $2 N$ is more decisive than the number of functions $2 n$. This is so because the number of functions $2 n$ increases the roundoff error (though to a certain degree it decreases the truncation error), while the number of boundary points $2 N$ refines the location of the maximum point in order to yield a more accurate value of the modulus $r$, see (4.23) and (4.24). Also, because the boundary is analytic, few functions are needed for the approximation.

Example 4. Let $D$ be a confocal elliptic ring, that is $D$ is given as in (6.7)-(6.9), but

$$
a_{1}^{2}-b_{1}^{2}=a_{2}^{2}-b_{2}^{2}
$$

In this case, the function

$$
f(z)=\frac{z+\left(z^{2}+b_{2}^{2}-a_{2}^{2}\right)^{1 / 2}}{a_{2}-b_{2}}
$$

maps $D$ onto the annulus $R$ in (3.1). The modulus of $D$ is given by

$$
r=\frac{a_{1}-b_{1}}{a_{2}-b_{2}}=\frac{a_{2}+b_{2}}{a_{1}+b_{1}}
$$

For $\left(a_{1}, b_{1}\right)=(11,9)$ and $\left(a_{2}, b_{2}\right)=(7,3)$, the modulus $r$ is $\frac{1}{2}$. The approximated modulus of $D$ is given in Table 4 . Here the moments are evaluated exactly as in Example 3. 
TABLE 4

\begin{tabular}{rcc}
\hline$n$ & $N$ & $r$ \\
\hline 8 & 5 & 0.48390541188 \\
8 & 9 & 0.49686407352 \\
12 & 5 & 0.48863028044 \\
12 & 9 & 0.49692203724 \\
\hline
\end{tabular}

Again, the pair $(n, N)=(12,9)$ in Table 4 gives the closest result to the theoretical value $r=0.5$.

Example 5. Let $D$ be a square of side $a$ inside a circle of radius $\rho$, that is

$$
D=\{z:|z|<\rho\} \cap\{(x, y):|x|>a / 2,|y|>a / 2\}, \quad \sqrt{ }(2 \rho)^{1 / 2}>a .
$$

Table 5 gives the approximated modulus of $D$, where $(\rho, a)=(7.7,10)$.

The moments are computed according to (5.2) and (5.7). $A_{11}$ is computed analytically while $A_{21}$ is approximated by the Gaussian quadrature rule ( $M=48$ points) (see [3], [5]).

TABLE 5

\begin{tabular}{rcc}
\hline$n$ & $N$ & $r$ \\
\hline 8 & 5 & 0.77692966148 \\
8 & 9 & 0.78102948051 \\
12 & 5 & 0.77929462853 \\
12 & 9 & 0.78114364275 \\
\hline
\end{tabular}

Again the theoretical value of $r$ is not known; however, we expect that the value 0.7811 gives a good approximation to the exact value of the modulus of $D$.

Table 6 gives the approximated modulus of $D$ where $(\rho, a)=(1,1)$.

TABLE 6

\begin{tabular}{rcc}
\hline$n$ & $N$ & $r$ \\
\hline 8 & 5 & 0.57924613312 \\
8 & 9 & 0.58296427281 \\
12 & 5 & 0.57963805403 \\
12 & 9 & 0.58302389835 \\
\hline
\end{tabular}

In [7, pp. 206-207 (Table 20)], the approximate modulus for this case is 0.583024 $(=1 / 1.715195)$. Thus, we expect that the value 0.583 is a good approximation of the modulus of $D$, where $(\rho, a)=(1,1)$. 
Example 6. Let $D$ be the domain bounded by two concentric squares of sides $a_{1}$ and $a_{2},\left(a_{2}>a_{1}\right)$, around the origin. For the pair $\left(a_{1}, a_{2}\right)=(a, 1), a<1$, the first seven orthonormal functions are given by

$$
\begin{aligned}
& P_{1}(z)=1 / 2\left(1-a^{2}\right)^{1 / 2}, \quad P_{2}(z)=\left(\sqrt{ } 3 / 2\left(1-a^{4}\right)^{1 / 2}\right) z, \\
& P_{3}(z)=\left((45)^{1 / 2} /\left(112\left(1-a^{6}\right)\right)^{1 / 2}\right) z^{2}, \\
& \begin{aligned}
P_{4}(z)= & \left(a / 2\left(\left(1-a^{2}\right)\left(1-a^{6}\right)\right)^{1 / 2}\right. \\
& \left.\cdot\left(98(2+\pi)\left(1-a^{6}\right)-1260(3-\pi)^{2} a^{2}\left(1-a^{2}\right)\right)^{1 / 2}\right) g(z), \\
& \quad g(z)=45(\pi-3)\left(1-a^{2}\right) z^{2}+28\left(1-a^{6}\right) z^{-2},
\end{aligned} \\
& P_{5}(z)=\left((35)^{1 / 2} /\left(96\left(1-a^{8}\right)\right)^{1 / 2}\right) z^{3}, \\
& P_{6}(z)=\left(4 a^{2} /\left((3 \pi+8)\left(1-a^{4}\right)\right)^{1 / 2}\right) z^{-3}, \\
& P_{7}(z)=\left(\sqrt{ } 7 /\left(1-a^{2}\right)^{1 / 2} \cdot\left(5312\left(1-a^{2}\right)\left(1-a^{10}\right)-448\left(1-a^{6}\right)^{2}\right)^{1 / 2}\right) f(z), \\
& \quad f(z)=4\left(1-a^{6}\right)+15\left(1-a^{2}\right) z^{4} .
\end{aligned}
$$

A more complicated expression is obtained for $P_{8}$. From these functions, we can form the kernel function and the conformal invariant $J_{D}(z)$ in (2.7), thus, we can determine the modulus of $D$; in particular, for the pair $(a, 1)=(0.2,1),(6.15)$ takes the form

$$
\begin{aligned}
& P_{1}(z)=0.5103103630798 \cdot z^{0}, \\
& P_{2}(z)=0.8667190566019 \cdot z^{1}, \\
& P_{3}(z)=0.6338859757222 \cdot z^{2}, \\
& P_{4}(z)=0.2783974154755 \cdot z^{2}+0.1274321780863 \cdot z^{-2}, \\
& P_{5}(z)=0.6038081372995 \cdot z^{3}, \\
& P_{6}(z)=0.3836049205974 \cdot z^{-3}, \\
& P_{7}(z)=0.1583600026160 \cdot z^{0}+0.5701324978974 \cdot z^{4} .
\end{aligned}
$$

In addition, we have

$$
\begin{aligned}
P_{8}(z)= & -0.2316641369540 \cdot z^{0} \\
& -0.7901077544546 \cdot z^{4}+0.1006646899038 \cdot z^{-4} .
\end{aligned}
$$

These functions were obtained by an exact integration of the corresponding moments, while most of our moments were computed by the Gaussian quadrature rule (48 points). Obviously, this integration is not exact for negative powers of $z$. However, it is exact for each polynomial of degree $\leqq 2.48-1=95$. Accordingly, the Gaussian rule yields the exact value of the polynomials $P_{1}(z), P_{2}(z), P_{3}(z), P_{5}(z), P_{7}(z)$ while it gives the approximated value of $P_{4}(z), P_{6}(z), P_{8}(z)$. Table 7 describes the modulus $r$ of $D$ for the pair $(0.2,1)$ achieved by the two methods of integration. Here, $2 n=8$, and we took $N=3$.

The exact value of $r$ is not known in this case. However, it is clear that since the number of the orthonormal functions is very small, the values of Table 7 give bad approximations to the modulus $r$. 
TABLE 7

\begin{tabular}{c|c|c|c}
\hline Integration & $n$ & $N$ & $r$ \\
\hline Analytic & 4 & 3 & 0.21471723637 \\
Gauss (48 Points) & 4 & 3 & 0.21471720316 \\
\hline
\end{tabular}

Table 8 gives the modulus $r$ of $D$ for the same pair $(0.2,1)$ where the Gaussian quadrature rule (48 points) is applied, but a larger number of orthonormal functions is used.

TABLE 8

\begin{tabular}{rcc}
\hline$n$ & $N$ & $r$ \\
\hline 8 & 3 & 0.49958185221 \\
8 & 5 & 0.51632857424 \\
16 & 3 & 0.49243158857 \\
16 & 5 & 0.50896817010 \\
\hline
\end{tabular}

From Table 8 we see that the value 0.509 gives a reasonable estimate for the modulus of $D$ with the pair $(0.2,1)$. The approximate modulus of $D$ in this case could also be computed by using differences (see [9]).

Appendix. We now prove the assertion made in the footnote of p. 748. For the sake of simplicity, let us assume that $C_{1}$ and $C_{2}$ are analytic curves. It will be clear that this assumption could be weakened considerably; for example, it is sufficient to require that $C_{1}$ and $C_{2}$ are piecewise analytic curves.

Suppose $D$ is in the $w$-plane and is the conformal image of the annulus $R=$ $\{z: r<|z|<1\}, 0<r<1$, by the conformal mapping $w=f(z)$. Each level line in $D$ is an image of a circle $|z|=\rho, r<\rho<1$. If $z=\rho e^{i \theta}$ describes this circle in the positive sense and its image is starshaped with respect to $w=0$, then the argument $\varphi$ of the image point $f(z)=\lambda e^{i \varphi}$ must vary in the same direction. Thus

$$
\partial \varphi / \partial \theta \geqq 0 \text {. }
$$

Conversely, if (A.1) holds, then the considered curve is starshaped. Equivalently, (A.1) can be written as

$$
\operatorname{Re}\left\{\frac{z f^{\prime}(z)}{f(z)}\right\} \geqq 0, \quad z=\rho e^{i \theta},
$$

$(r<\rho<1)$, and (A.2) is a necessary and sufficient condition for the level curve to be starshaped.

By assumption, $C_{v}(\nu=1,2)$ are analytic, therefore, $w=f(z)$ is conformal from $\bar{R}=\{z: r \leqq|z| \leqq 1\}$ onto the closure of $D, \bar{D}$. Consequently, if $C_{\nu}(\nu=1,2)$ are starshaped with respect to $w=0$, then (A.2) holds for $\rho=r$, 1 . Since $0 \notin \bar{D}$, it follows that $z f^{\prime}(z) / f(z)$ is analytic on $\bar{R}$, and so $\operatorname{Re}\left\{z f^{\prime}(z) / f(z)\right\}$ is harmonic on $\bar{R}$. 
By the minimum principle for harmonic functions, (A.2) holds throughout $\bar{R}$, and, hence, each level curve in $\bar{D}$ is starshaped with respect to $w=0$.

Acknowledgment. The author wishes to thank Professor P. Rabinowitz, Weizmann Institute of Science, for his valuable help. In addition, the author would like to thank the referee for his invaluable comments.

Department of Mathematics

Stanford University

Stanford, California 94305

1. S. Bergman, The Kernel Function and Conformal Mapping, 2nd ed., Math. Surveys, no. 5, Amer. Math. Soc., Providence, R.I., 1970.

2. S. Bergman \& B. Chalmers, "A procedure for conformal mapping of triply-connected domains," Math. Comp., v. 21, 1967, pp. 527-542. MR 37 \#4243a.

3. J. BuRBEA, "A procedure for conformal maps of simply connected domains by using the Bergman function," Math. Comp., v. 24, 1970, pp. 821-829.

4. J. Burbea, "On the Gaussian curvature related to the Szegö metric." (To appear.)

5. P. Davis \& P. Rabinowitz, "Abscissas and weights for Gaussian quadratures of high order," J. Res. Nat. Bur. Standards, Sect. B, v. 56, 1965, pp. 35-37.

6. B. EpsteIN, Orthogonal Families of Analytic Functions, Macmillan, New York, 1965. MR 31 \#4916.

7. D. GaIER, Konstruktive Methoden der konformen Abbildung, Springer Tracts in Natural Philosophy, vol. 3, Springer-Verlag, Berlin, 1964. MR 33 \#7507.

8. J. KIEFER, "Sequential minimax search for a maximum," Proc. Amer. Math. Soc., v. 4, 1953, pp. 502-506. MR 14, 1103.

9. G. OPFER, "Die Bestimmung des Moduls zweifach zusammenhängender Gebiete mit Hilfe von Differenzenverfahren," Arch. Rational Mech. Anal., v. 32, 1969, pp. 281-297. MR 38 \#4662.

10. M. C. Pike \& J. Pixner, "Fibonacci search," Comput. Bull., v. 8, 1965, p. 147.

11. P. Rabinowitz, "Numerical experiments in conformal mapping by the method of orthonormal polynomials," J. Assoc. Comput. Mach., v. 13, 1966, pp. 296-303.

12. K. ZaRANKIEWICZ, "Über ein numerisches Verfahren zur konformen Abbildung zweifach zusammenhängender Gebiete," Z. Angew. Math. Mech., v. 14, 1934, pp. 97-104. 